\title{
Linear and Nonlinear Active Disturbance Rejection Controllers for Single-Link Flexible Joint Robot Manipulator based on PSO Tuner
}

\author{
Amjad J. Humaidi ${ }^{1, *}$ and Hussein M. Badr ${ }^{2}$ \\ ${ }^{l}$ University of Technology, Control and Systems Engineering Department, Iraq-Baghdad \\ ${ }^{2}$ Ministry of Electricity, Training and Energy Researches Office, Iraq-Baghdad
}

Received 11 December 2017; Accepted 2 August 2018

\begin{abstract}
This work presents the design of active disturbance rejection control (ADRC) strategy to position control of a single-link flexible joint robot manipulator. Two schemes of ADRC are presented, Linear Active Disturbance Rejection Controller (LADRC) and Nonlinear Active Disturbance Rejection Controller (NADRC). A comparison study of transient performances, robustness characteristics and disturbance rejection capabilities has been made based on LADRC and NADRC. The robustness of both controllers is evaluated by measuring the amount of deviation is system performance due to uncertainty in system parameters, while the disturbance rejection capability of both controllers will be investigated under abrupt disturbance exertion. One issue with NADRC and LADRC is they include various parameters and unless they are properly tuned, will have an adverse effect on the estimation process and in turn, on the system performance. The particle swarm technique (PSO) has been selected as an optimal tuner to improve the estimation process and thereby, to enhance the system performance.
\end{abstract}

Keywords: Flexible Joint Robot, LADRC, NADRC, PSO.

\section{Introduction}

In flexible joint robot manipulators, the elasticity of the transmission devices on the joints has been taken into account. Considering the effect of joint flexibility would develop a more exact model of industrial robot. However, a further complexity is added to the robot model, which raises a new control problem and therefore motivated the researchers in this relevant field further indulge.

In the past three decades many researchers have worked in the control of flexible joint robots. In [1], the sliding mode control strategy has been applied to a flexible joint manipulator. However, for feasible implementation of this control scheme, the control design requires the knowledge of uncertainty bounds and also the complete information of all system states. In [2], a dynamic feedback control design is developed for the trajectory tracking control problem of robotic manipulators with flexible joints. In this control design, the position measurements of the link and motor are needed, such that a reduced-order observer is used to estimate the required velocities for control. However, in this work, the establishment of system robustness requires certain conditions to be imposed on the uncertainties. In [3], the proposed control is based on a singular perturbation approach and the measurements of elastic force and position are prerequisites for the control design. This work suggested a nonlinear sliding state observer to estimate link velocities and elastic force time derivatives required for this control strategy. A control design based on the integral manifold formulation [4], adaptive control [5] and a back-

*E-mail address: 601116@uotechnology.edu.iq

ISSN: 1791-2377 @ 2018 Eastern Macedonia and Thrace Institute of Technology. All rights reserved. doi:10.25103/jestr.113.18 stepping approach [6] are some other approaches reported in the literature. It has been seen that the majority of control designs in the literature require either information of complete states or at least the position state or some state on the motor side. Moreover, the guarantee of robustness may be highly dependent on the systems model and requires the knowledge of some characteristics of uncertainties.

Active Disturbance Rejection Controller (ADRC) was firstly proposed by J. Han with nonlinear gains. The control design of ADRC focused on nonlinear systems and considered both uncertain dynamics and disturbances. The essential idea of ADRC is firstly to combine both internal uncertain dynamics and external disturbances into a total uncertainty and to then estimate this combined uncertainty by an extended state observer (ESO) and thereafter to be cancelled out using state feedback structure. This renders ADRC applicable for many practical systems [7]. The Nonlinear ADRC (NADRC) has been parameterized and modified to linear ADRC (LADRC) with linearized gains by Z. Gao [8]. ADRC requires little information of the plant and is not completely dependent on the mathematical model of the system, which makes it very robust against system uncertainty [7].

Active Disturbance Rejection Control (ADRC) has recently attracted the interest of many researchers in robotics. A method using ADRC has been proposed for the control of a flexible joint robot in the control design, using cascade Nonlinear ADRC [9] and a feedback linearization (FL) based control law based upon ESO is presented in [10].

In the present work, two active disturbance rejection control schemes are presented, NADRC and LADRC, in order to control a single-link flexible joint robot manipulator; the work contribution can be highlighted by 
the following points:

(1) A performance comparison between linear and Nonlinear ADRC is made in terms of dynamic behavior and robustness capability.

(2) The suggested controls have to control the nonlinear system structure of the flexible joint manipulator without linearization.

(3) A second order ADRC has been used to control a fourth order system.

(4) The PSO technique is included to improve the closed-loop system performance.

\section{Joint Flexibility and System Modeling}

The modeling, simulation and real-time control of flexible manipulators is still challenging and remains an open problem. Many academic and industrial researchers have considered flexibility at joints due to their significant effect and it may dominate over link flexibility of the majority of manipulators available nowadays. This is evident in many applications where industrial robotic manipulators are driven by harmonic drive gears [11].

The flexibility at a joint can be modelled by a linear, torsional spring as indicated in Fig. (1). It is clear that a motor drives the arm via a spring, attached to the motor shaft [12].

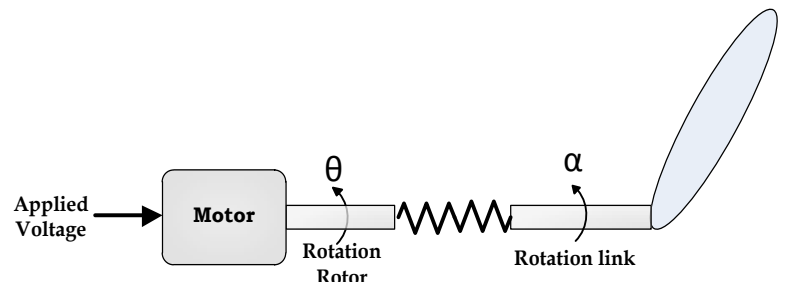

Fig. 1. Flexible joint robot.

The modeling of a single-link, flexible joint robot is considered, operating on a vertical plane and developed using the Lagrange method. The method is based on conservation of energy and is initiated by calculating the difference between kinetic energy $\mathrm{K}$ and the potential energy $\mathrm{P}$ of all system components [12]

$$
L=K-P
$$

Lagrange equations are configured as follows:

$$
\begin{aligned}
& \frac{d}{d t} \frac{d L}{d \dot{\alpha}}-\frac{d L}{d \alpha}=0 \\
& \frac{d}{d t} \frac{d L}{d \dot{\theta}}-\frac{d L}{d \theta}=\tau
\end{aligned}
$$

Based on Fig. (1), the following equations can be derived [12]:

$$
\begin{aligned}
& J_{l} \ddot{\theta}+J_{l} \ddot{\alpha}+K_{s} \alpha-m g h \sin (\theta+\alpha)=0 \\
& \left(J_{h}+J_{l}\right) \ddot{\theta}+J_{l} \ddot{\alpha}-m g h \sin (\theta+\alpha)=\tau
\end{aligned}
$$

Using Kirchhoff's Voltage Law (KVL) and neglecting the motor inductance, the electrical side of the system gives:

$v=i R_{m}+K_{m} K_{g} \omega$

$i=\left(v / R_{m}\right)-\left(K_{m} K_{g} \omega / R_{m}\right)$
Where $\omega$ is the angular velocity of the motor $(\dot{\theta}=\omega)$, v is the applied input voltage, $R_{m}$ is the armature resistance, $\mathrm{K}_{\mathrm{m}}$, motor gain and $\mathrm{K}_{\mathrm{g}}$ is the gear ratio. The current is related to developed torque $\tau$ by the following equation:

$\tau=K_{m} K_{g} i$

One can then deduce from Eq. (7) and (6) that:

$\tau=K_{m} K_{g}\left(v / R_{m}\right)-\left(K_{m}^{2} K_{g}^{2} \omega / R_{m}\right)$

Substituting Eq. (4) into Eq. (5) yields

$J_{h} \ddot{\theta}-K_{s} \alpha=K_{m} K_{g} v / R_{m}+K_{m}^{2} K_{g}^{2} \omega / R_{m}$

or,

$\ddot{\theta}=\left(K_{m} K_{g} / J_{h} R_{m}\right) v-\left(K_{m}^{2} K_{g}^{2} / J_{h} R_{m}\right) \dot{\theta}+\left(\frac{K_{s}}{J_{h}}\right) \alpha$

From Eq. (9) and Eq. (4), one can get

$$
\begin{aligned}
\ddot{\alpha}=-\left(K_{m} K_{g} / J_{h}\right. & \left.R_{m}\right) v+\left(K_{m}^{2} K_{g}^{2} / J_{h} R_{m}\right) \dot{\theta}-\left(K_{s} / J_{h}\right) \alpha \\
& -\left(K_{s} / J_{l}\right) \alpha \\
& +\left(m g h / J_{l}\right) \sin (\theta+\alpha)
\end{aligned}
$$

The arm angle (tip angle) is composed of the sum $\alpha$ and $\theta$. Letting $x_{1}=\theta, x_{2}=\alpha, x_{3}=\dot{x}_{1}=\dot{\theta}, x_{4}=\dot{x}_{2}=\dot{\alpha}$, then the system described by Eq. (9) and Eq. (10) can be written in state variable form [14]:

$$
\begin{aligned}
& \dot{x_{1}}=x_{3} \\
& \dot{x_{2}}=x_{3} \\
& \dot{x_{3}}=\left(K_{m} K_{g} / J_{h} R_{m}\right) v-\left(K_{m}^{2} K_{g}^{2} / J_{h} R_{m}\right) x_{3} \\
& +\left(K_{s} / J_{h}\right) x_{2} \\
& \dot{x_{4}}=-\left(K_{m} K_{g} / J_{h} R_{m}\right) v+\left(K_{m}^{2} K_{g}^{2} / J_{h} R_{m}\right) x_{3} \\
& -\left(K_{s} / J_{h}\right) x_{2}-\left(K_{s} / J_{l}\right) x_{2}+\left(m g h / J_{l}\right) \sin \left(x_{1}+x_{2}\right) \\
& y=x_{1}+x_{2}
\end{aligned}
$$

\section{Active Disturbance Rejection Control}

\subsection{Structure of the Nonlinear ADRC}

The Nonlinear ADRC control consists of two primary parts; the Tracking Differentiator (TD) and the Nonlinear Extended State Observer (NESO). The tracking differentiator's function is to manage the transient process and in addition, if the input is corrupted by noise, then appropriate filters and a tracking differentiator are used to remove the noise effect. The mathematical description of TD is generally given by [13]:

$$
\begin{aligned}
& \dot{z}_{1}=z_{2} \\
& \dot{z}_{2}=f_{h} \\
& f_{h}=f_{\text {han }}\left(z_{1}-v, z_{2}, r, h\right)
\end{aligned}
$$


Where $v$ is the input signal, $r$ is called the speed factor, $h$ is the filter factor and $f_{\text {han }}\left(x_{1}, x_{2}, r, h\right)$ is the integrated function of time optimum control, which is derived by:

$f_{\text {han }}= \begin{cases}r \cdot \operatorname{sign}(a) & |y|>d \\ r \frac{a}{d} & |y| \leq d\end{cases}$

Where,

$a= \begin{cases}x_{1}+\frac{(a o-d)}{2} \operatorname{sign}(y), & |y|>d o \\ x_{2}+\frac{y}{h}, & |y| \leq d o\end{cases}$

$d=r . h$

$d_{o}=h \cdot d$

$y=x_{1}+x_{2} \cdot h$

$a_{o}=\sqrt{d^{2}+8 \cdot r \cdot|y|}$

For the position control of single-link flexible joint robot manipulator, the angle of flexible joint robot, $z_{1}$, track the angle of flexible joint robot, $z_{2}$, track the differential signal.

The second element of Nonlinear ADRC is Nonlinear Extended State Observer (NESO). This structure of observer was proposed by J. Han in 1995 and characterized by independency of a plant mathematical model, thus achieving inherent robustness. The descriptive model is given by:

$$
\begin{aligned}
& e=z_{1}-y \\
& \dot{z}_{1}=z_{2}-L_{1} \cdot e \\
& \dot{z}_{2}=z_{3}-L_{2} \cdot f a l\left(e, \alpha_{1}, \delta_{1}\right)+b . u \\
& \dot{z}_{3}=-L_{3} \cdot f a l\left(e, \alpha_{2}, \delta_{2}\right)
\end{aligned}
$$

Where $e$ is the error between actual and estimated output of system, $L_{1}, L_{2}, L_{3}$ represent the gains of observer, $z_{1}, z_{2}$ are the estimates of the states $x_{1}$ and $x_{2}$. The state variable $z_{3}$ is the estimate state of combined uncertain and nonlinearity disturbances (external and internal) of the system. The function $\operatorname{fal}(e, \alpha, \delta)$ is a nonlinear function:

$$
f a l(e, \alpha, \delta)=\left\{\begin{array}{c}
\frac{e}{\delta^{\alpha-1}},|e| \leq \delta \\
|e|^{\alpha} \operatorname{sign}(e),|e|>\delta
\end{array}\right.
$$

Which yields high gain when error is small, $\alpha$ is chosen between ( 0 and 1$), \delta$ is a small number used to limit the gain in the neighbourhood of origin.

The third element of NADRC is the Nonlinear State Error Feedback (NLSEF). The mathematical structure of NLSEF is written by:

$$
\begin{aligned}
& e_{1}=v_{1}-z_{1} \\
& e_{2}=v_{2}-z_{2} \\
& u_{o}=k_{p} \cdot \operatorname{fal}\left(e_{1}, \alpha_{1}, \delta_{1}\right)+k_{d} \cdot \operatorname{fal}\left(e_{2}, \alpha_{2}, \delta_{2}\right) \\
& u=u_{o}-\frac{z_{3}}{b}
\end{aligned}
$$

Where $e_{1}, e_{2}$ represent the error between input signals and the estimate states of system, $k_{p}, k_{d}$ are gains of NLSEF, $f a l\left(e_{1}, \alpha_{1}, \delta_{1}\right), f a l\left(e_{2}, \alpha_{2}, \delta_{2}\right)$ are the nonlinear functions of NLSEF.

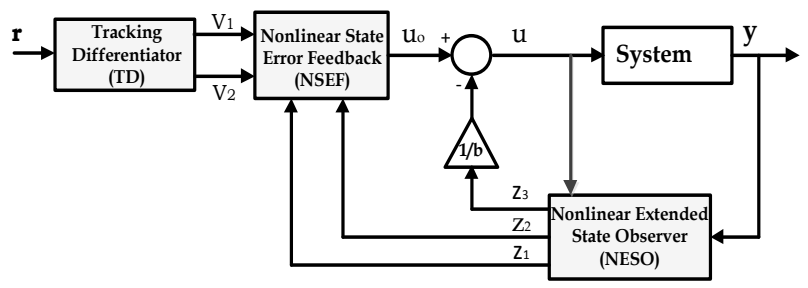

Fig. 2. A schematic representation of NADRC.

\subsection{Structure of the Linear ADRC}

The structure of the LADRC control is composed of ESO (Extended State Observer) and SEF (State Error Feedback). The tracking differentiator has been omitted in the present structure and due to simplicity in parameter tuning, the Linear Extended State Observer (LESO) has been used, which is a linear version of Nonlinear Extended State Observer (NESO). The mathematical structure of LESO can be described by the following:

$e=y-z_{1}$

$\dot{z}_{1}=z_{2}+L_{1} \cdot e$

$\dot{z_{2}}=z_{3}+L_{2} \cdot e+b \cdot u$

$\dot{z_{3}}=L_{3} \cdot e$

Where, $L_{1}, L_{2}$ and $L_{3}$ are the observer gains and the variable $z_{3}$ stands for lumped disturbance and uncertainty, together with system nonlinearity. The second element of LARC is LSEF, which can be represented by the following set of equations:

$e_{1}=v_{1}-z_{1}$

$e_{2}=v_{2}-z_{2}$

$u_{o}=k_{p} \cdot e_{1}+k_{d} \cdot e_{2}$

$u=u_{o}-z_{3} / b$

Where $e_{1}, e_{2}$ represent the error between input signals and estimated states of system and $k_{p}, k_{d}$ are the gains of linear state feedback (LSEF). Fig. (4) shows the schematic diagram of Linear ADRC.

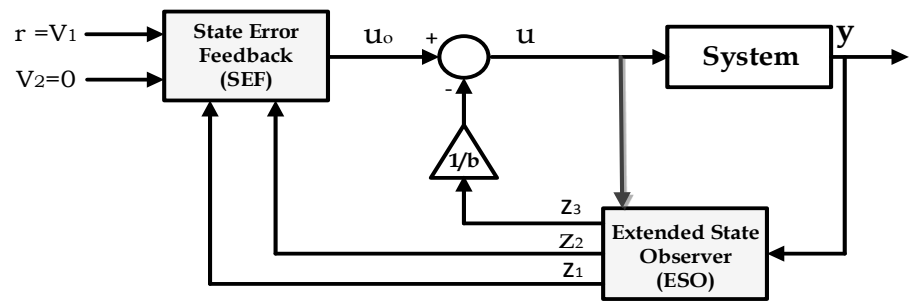

Fig. 3. A schematic representation of LADRC

\subsection{ADRC-Based on PSO}

The original ADRC system possesses many parameters, which are required to be tuned to improve the control capabilities. Due to the complexity of ADRC and tight coupling of its constituting parameters, the trial-and-error 
tuning process is exhaustive and does not lead to global optimizing solutions and as such, an alternative optimization technique is required. In the present work, a Particle Swarm Optimization (PSO) technique has been selected for tuning purposes. The PSO algorithm is based on the behavior of individuals of swarm. These individuals share information among themselves and this leads to increased efficiency of the group [14]. This optimization method will not only responsible for the tuning of all parameters but also to establish the best value for it, which will achieve minimum error between desired and actual states or between the inputs and outputs of the system.

Using the PSO technique, rather than trial-and-error methods will improve the estimation process and hence the closed-loop system dynamic is better enhanced, due to improved estimates of the observer. It is evident that the number of parameters associated with LADRC are much less than that of NADRC. The numerical values presented in the above tables represent the optimal values of parameters, which are tuned based on estimation error minimization. The fitness function composed by the RMSE (Root Mean Square Error) index is adopted in comprehensive consideration of the rapidity, stability and accuracy of the system.

$J(R M S E)=\sqrt{\frac{\sum_{i=1}^{n} e^{2}}{n}}$

A parameterization method proposed by Gao [8] is used to tune the parameters of LADRC. This parameterization method determines the gains value of both controller and observer, based on bandwidth of the observer $\omega_{0}$ and control $\omega_{c}$. This can be clarified using the relationship between controller gains and its bandwidth frequency $\omega_{c}$ :

$\lambda(s)=s^{2}+k_{d} s+k_{p}=\left(s+\omega_{c}\right)^{2}$

where, $k_{d}=2 \omega_{c}, \quad k_{p}=\omega_{c}{ }^{2}$. Similarly, based on the following equation, which relates observer gains and its bandwidth, the observer gains can be obtained:

$\lambda(s)=s^{3}+L_{1} s^{2}+L_{2} s+L_{3}=\left(s+\omega_{0}\right)^{3}$

Where, $L_{1}=3 \omega_{0}, L_{2}=3 \omega_{0}^{2}, L_{3}=\omega_{0}^{3}$. Thus, this method could simplify the level of complexity to determine the optimal gain values of LESO and NESO, based on PSO. Table 1 lists the optimal parameters of NESO, NLSEF and TD, which have been tuned by the PSO algorithm. Alternately, Table 1 shows the optimal parameters of LESO and LSEF, resulting from the PSO optimizer.

Table 1. Optimal parameters of NADRC

\begin{tabular}{|c|c|c|c|c|c|}
\hline $\begin{array}{l}\text { NESO. } \\
\text { Param. }\end{array}$ & Value & $\begin{array}{l}\text { NLSEF. } \\
\text { Param. }\end{array}$ & Value & $\begin{array}{c}\text { TD. } \\
\text { Param. }\end{array}$ & Value \\
\hline$L_{1}$ & 53.9 & $\beta_{1}$ & 3.2324 & $h$ & 0.000001 \\
\hline$L_{2}$ & 969.7 & $\beta_{2}$ & 3.5958 & & \\
\hline$L_{3}$ & 5811.6 & $\alpha_{1}$ & 0.9 & & \\
\hline$b$ & 46.3634 & $\delta_{1}$ & 0.00001 & & \\
\hline$\alpha_{1}$ & 1 & $\alpha_{2}$ & 1.25 & & \\
\hline$\delta_{1}$ & 0.1 & $\delta_{2}$ & 0.00001 & & \\
\hline$\alpha_{2}$ & 0.98 & & & & \\
\hline$\delta_{2}$ & 0.1 & & & & \\
\hline$\alpha_{3}$ & 0.78 & & & & \\
\hline$\delta_{3}$ & 0.1 & & & & \\
\hline
\end{tabular}

Table 2. Optimal parameters of LADRC

\begin{tabular}{|c|c|c|c|}
\hline LESO. Param. & Value & LSEF. Param. & Value \\
\hline$\omega_{0}$ & 17.979 & $\omega_{c}$ & 1.7979 \\
\hline$L_{1}$ & 53.9 & $\beta_{1}$ & 3.2324 \\
\hline$L_{2}$ & 969.7 & $\beta_{2}$ & 3.5958 \\
\hline$L_{3}$ & 5811.6 & \multicolumn{3}{|}{} \\
\hline$b$ & 46.3634 & &
\end{tabular}

\section{Simulated Results}

In this section, the dynamic behavior of a flexible-joint system, based on ADRC, is established using MATLAB/Simulink. The numeric values of system parameters are listed in Table 3. The first comparison is made based on how well the transient characteristics could be given by such controllers. The controller reference input is assigned to be a step input of height $30^{\circ}$ and the tip angle $(\theta+\alpha)$ is the output response, which have to be controlled. Fig. (4) shows the dynamic behaviors of a flexible-joint system resulting from both controllers. The figure shows that NADRC offer better transient characteristics than LADRC. Table (4) makes a quantitative comparison based on simulation, which confirms that the response due to NLADRC outperforms the response resulting from LADRC. The key index of comparison is measured by root means square error (RMSE), which calculate the root mean square of error over the entire response. The response with less RMSE will address the best controller.

Table 3. Numerical values of system parameters [12]

\begin{tabular}{c|c|c}
\hline Parameter & Symbol & Value \\
\hline Load Inertia & $J_{l}$ & $0.0059\left[\mathrm{~kg} . \mathrm{m}^{2}\right]$ \\
Inertia of hub & $J_{h}$ & $0.0021\left[\mathrm{~kg} . \mathrm{m}^{2}\right]$ \\
Link Mass & $m$ & $0.403[\mathrm{~kg}]$ \\
Height of C.M. & $h$ & $0.06[\mathrm{~m}]$ \\
Spring Stiffness & $K_{s}$ & $1.61[\mathrm{~N} / \mathrm{m}]$ \\
\hline Motor Const. & $K_{m}$ & 0.00767 \\
Gear Ratio & $K_{g}$ & 70 \\
Motor Resist. & $R_{m}$ & $2.6[\Omega]$ \\
Gravity Const. & $\mathrm{g}$ & $-9.81[\mathrm{~N} / \mathrm{m}]$ \\
\hline \multicolumn{3}{|c}{ Arm angle } \\
\hline
\end{tabular}
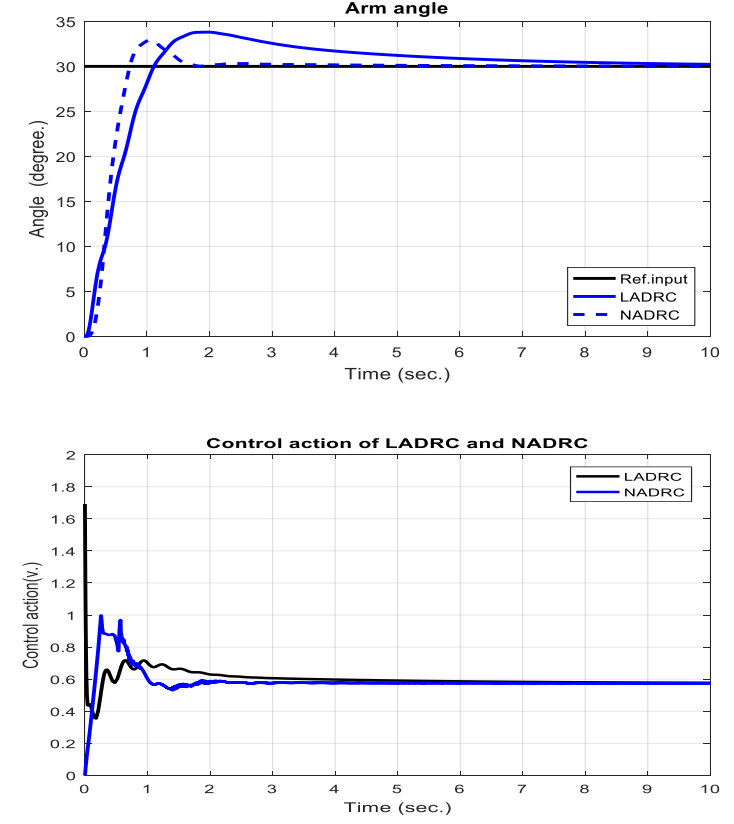

Fig. 4. Performance of NADRC and LADRC with nominal case 
Table 4. Dynamic performance report of controllers

\begin{tabular}{c|c|c|c}
\hline Controller & RMSE & $\begin{array}{c}\text { Maximum } \\
\text { overshoot \% }\end{array}$ & $\begin{array}{c}\text { Settling Time } \\
\text { (sec.) }\end{array}$ \\
\hline LADRC & 9.7576 & 3.8 & 7.133 \\
NADRC & 2.9558 & 2.93 & 1.54 \\
\hline
\end{tabular}

Another comparison is made to assess the robustness capabilities of the suggested controllers due to changes of system parameters. Two uncertainties are permitted to appear in the present work; internal uncertainty, represented by changing link mass and center of mass and external uncertainty given by disturbance exertion. Fig. (5) and Fig. (6) show the dynamic responses of tip angle and control signals resulting from LADRC and NLADRC, respectively. The responses indicated in the figures correspond to three values of masses (nominal value of mass, two times nominal value of mass, three times nominal value of mass). On the other hand, Fig. (7) and Fig. (8) show the transient responses of tip angle and control signals due to LADRC and NLADRC, respectively, and under a change of mass center $(h)$. In this scenario, two cases of mass centers have been addressed; the nominal value and $10 \%$ deviated value (from the nominal).

The next simulations are dedicated for evaluation of disturbance rejection capability of both controllers. The disturbance is injected as angle perturbation between time 7-7.2 seconds with height of $20^{\circ}$. The performances of LADRC and NLADRC under this prescribed disturbance application have been illustrated in Fig. (9) and Fig. (10), respectively.
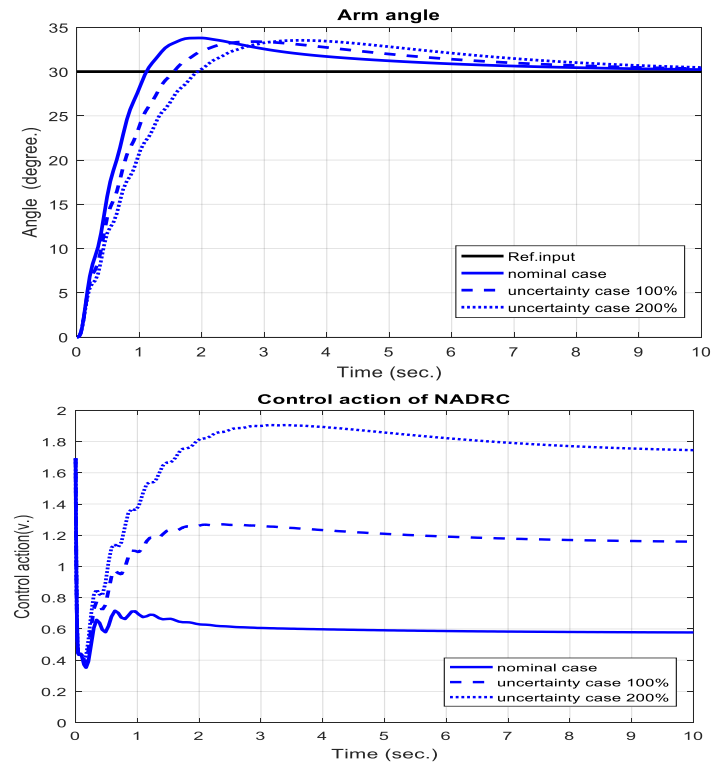

Fig. 5. Dynamic responses of LADRC with three different settings of mass

Table (5) gives a summary of robustness characteristics for both controllers. The table reports the deviation in transient response from the nominal case, measured in RMSE, when the system is subjected to parameter variations (change in mass, change in the length of C.M). It is evident from the table that the minimum deviation from the nominal occurs with LADRC and this indicates that this controller have better robustness characteristics than NLADRC. In Table (5), the disturbance rejection capability of both controllers are evaluated in terms of how the response has been deviated upon abrupt disturbance change. RMSE measure is also used here to quantify the deviation. It is clear from the table that LADRC has better disturbance rejection capability than its opponent.
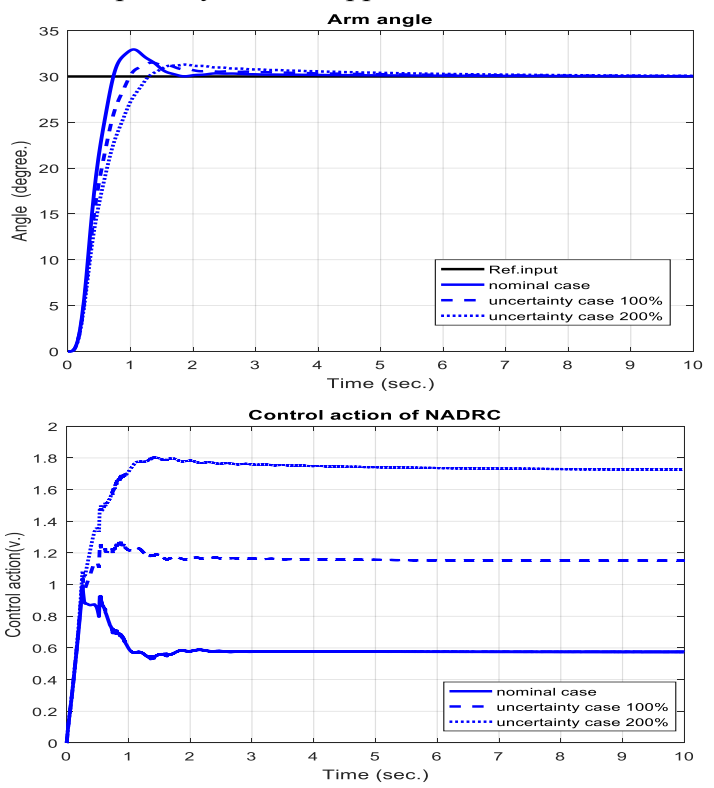

Fig. 6 Dynamic responses of NADRC with three different setting of mass
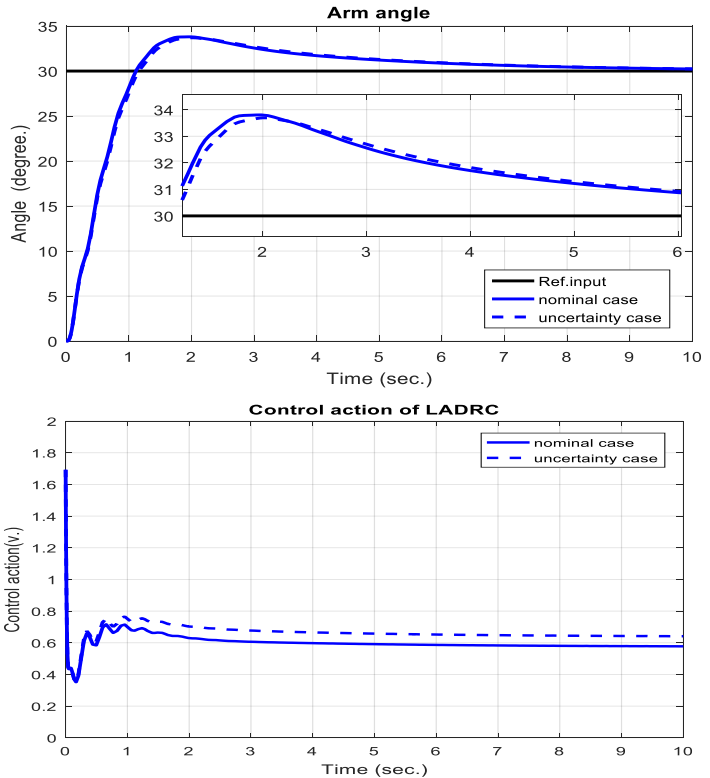

Fig. 7 Dynamic responses of LADRC with two different setting of link length
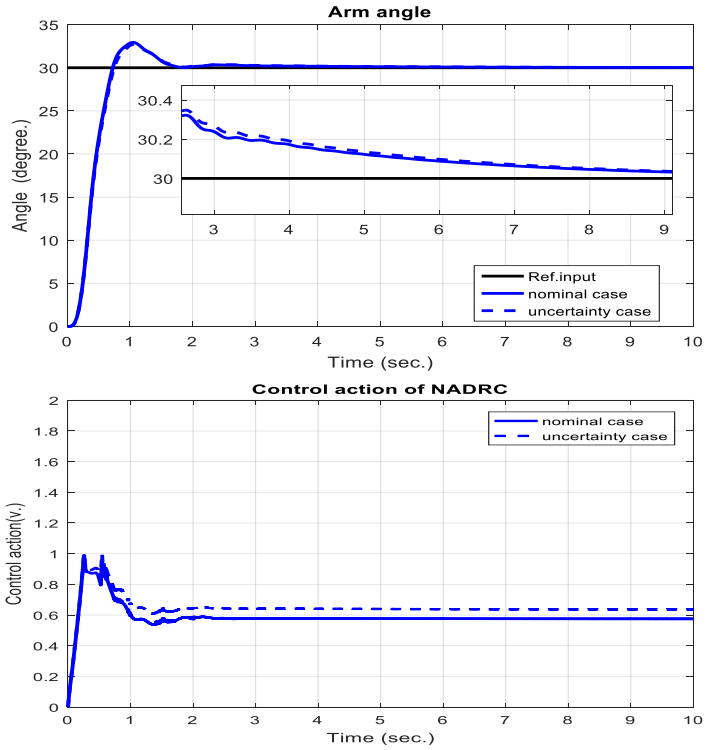

Fig. 8. Dynamic responses of LADRC with two different setting of link 
length
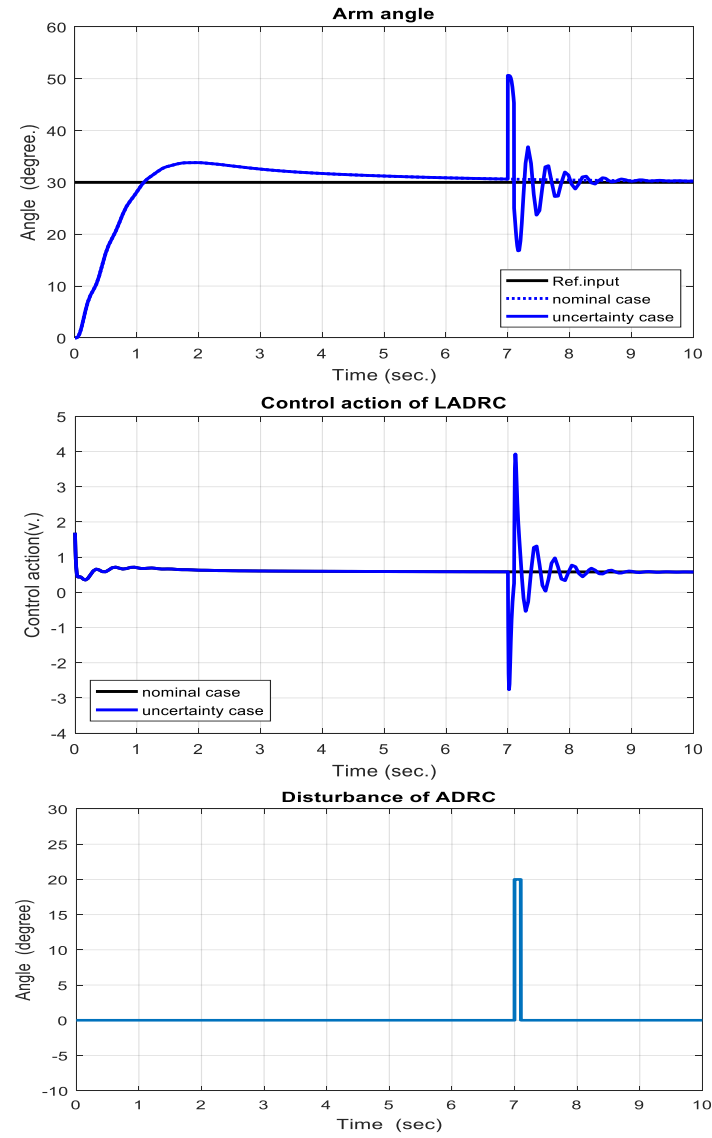

Fig. 9 Dynamic responses of LADRC with disturbance

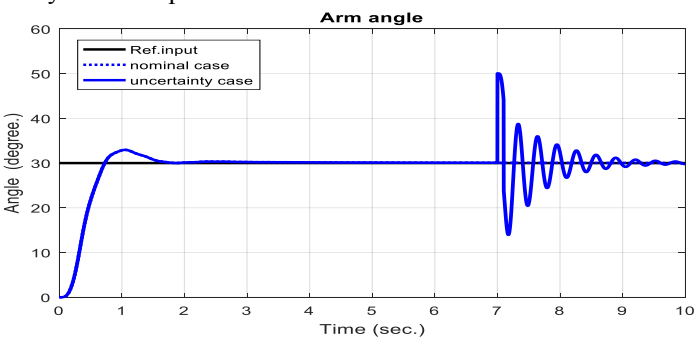

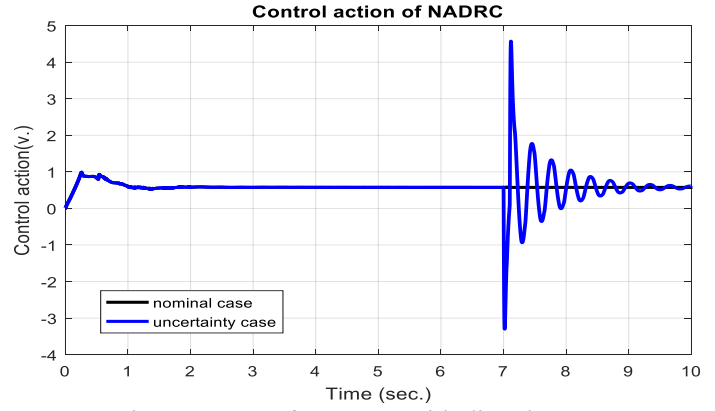

Fig. 10 Dynamic responses of NADRC with disturbance

Table 5. Robustness evaluation of controllers

\begin{tabular}{c|c|c}
\hline \multirow{2}{*}{$\begin{array}{c}\text { Percentage change of } \\
\text { system parameters }\end{array}$} & \multicolumn{2}{|c}{$\begin{array}{c}\text { Percentage Change from } \\
\text { nominal }\end{array}$} \\
\cline { 2 - 3 } & LADRC & NADRC \\
\hline$+100 \%$ of nominal mass & $2.9444 \%$ & $10.9683 \%$ \\
$+200 \%$ of nominal mass & $6.6348 \%$ & $32.9454 \%$ \\
$+10 \%$ of nominal C.M & $0.3105 \%$ & $2.5746 \%$ \\
Disturbance Application & $7.6156 \%$ & $47.1344 \%$ \\
\hline
\end{tabular}

\section{Conclusion}

This paper presents a comparison study between two active rejection disturbance controllers in terms of transient characteristics and robustness. Based on simulated results, one can conclude that NLADRC offer better dynamic behavior than LADRC under nominal condition. However, LADRC possesses better robustness characteristics and disturbance rejection capabilities than NLADRC when the system is subjected to a variation of parameters and sudden change in disturbance. PSO has played a vital role in optimization and performance enhancement of an overall active rejection disturbance controlled system.

This is an Open Access article distributed under the terms of the Creative Commons Attribution License

\section{(1) $\$$}

\section{References}

[1] S. K. Spurgeon, L. Yao, and X. Y. Lu, "Robust tracking via sliding mode control for elastic joint manipulators," Proc. Inst. Mech. Eng. I, vol. 215, no. 4, pp. 405-417, 2001.

[2] Y. C. Chang, B. S. Chen, and T. C. Lee, "Tracking control of flexible joint manipulators using only position measurements," Int. J. Control, vol. 64, no. 4, pp. 567-593, 1996.

[3] J. Hernandez and J.-P. Barbot, "Sliding observer-based feedback control for flexible joints manipulator," Automatica, vol. 32, no. 9, pp. 1243-1254, Sep. 1996.

[4] M. W. Spong, "Modeling and control of elastic joint robots," Trans. ASME, J. Dyn. Syst., Meas., Control, vol. 109, no. 4, pp. 310319, Dec. 1987.

[5] F. Ghorbel, J. Y. Hung, and M. W. Spong, "Adaptive control of flexible joint manipulators," IEEE Control Syst. Mag., vol. 9, no. 7, pp. 9-13, Dec. 1989.

[6] J. Oh and J. Lee, "Control of Flexible Joint Robot System by Backstepping Design Approach," Intelligent Automation \& Soft Computing, vol. 5, no. 4, pp. 267-278, 1999.

[7] J. Han, "From PID to Active Disturbance Rejection Control", IEEE Transactions on Industrial Electronics, vol. 56, no. 3, pp. 900906, 2009.

[8] Z. Gao, "Scaling and bandwidth-parameterization based controller tuning", Proceedings of the 2003 American Control Conference, 2003., vol. 6, no. 7, pp. 4989 - 4996, 2003.
[9] Y. Zhao, Z. Zhao, B. Zhao and W. Li, "Active Disturbance Rejection Control for Manipulator Flexible Joint with Dynamic Friction and Uncertainties Compensation", Fourth International Symposium on Computational Intelligence and Design, vol. 2, no. 1, pp. $248-251,2011$.

[10] S. Talole, J. Kolhe and S. Phadke, "Extended-State-ObserverBased Control of Flexible-Joint System with Experimental Validation", IEEE Transactions on Industrial Electronics, vol. 57, no. 4, pp. 1411-1419, 2010.

[11] B. Siciliano and O. Khatib, Springer handbook of robotics. Berlin: Springer, pp. 287-295, 2008.

[12] A. Dehghani and H. Khodadadi, "Self-Tuning PID Controller Design Using Fuzzy Logic for Single-Link Flexible Joint Robot Manipulator", Jurnal Teknologi, vol. 78, no. 6-13, 2016.

[13] F. Meng, J. Yang, P. Yang and B. Sun, "The Research of Frequency Characteristics of Tracking Differentiator", Applied Mechanics and Materials, vol. 182-183, pp. 1474-1478, 2012.

[14] Z. Gaing, "A Particle Swarm Optimization Approach for Optimum Design of PID Controller in AVR System", IEEE Transactions on Energy Conversion, vol. 19, No. 2, pp. 384-391, 2004. 